(C) Masson, Paris, 1980 .

\title{
Artemia sp. (Crustacé, Anostracé), hôte intermédiaire d'Eurycestus avoceti Clark, 1954 (Cestode Cyclophyllide)
}

\author{
parasite de l'avocette en Camargue. \\ par C. GABRION * et G. MAC DONALD **
}

* Laboratoire de Parasitologie comparée, U.S.T.L., Place E.-Bataillon, F 34060 Montpellier. *" Station Biologique de la Tour du Valat, Le Sambuc, F 13200 Arles.

RESUME. Au cours d'une étude sur les populations d'Artemia sp. (Crustacé, Anostracé) de Camargue, infestées naturellement par des cysticercoïdes de Flamingolepis liguloides (Cestode, Cyclophyllide) parasite du Flamant (Phoenicopterus ruber), les auteurs observent, outre $F$. liguloides, la présence de quatre autres cysticercoïdes. Trois d'entre eux appartiennent à des Cestodes du Flamant. Par contre, le quatrième est le cysticercoïde d'un Cestode de l'Avocette (Recurvirostra avosetta) Eurycestus avoceti Clark, 1954, dont la position systématique reste problématique.

Artemia sp. (Crustacea, Anostracea) as intermediate host of Eurycestus avoceti Clark, 1954 (Cestoda, Cyclophyllidea).

SUMMARY. Examination of Artemia sp. (Crustacé, Anostracé) for natural infection by cysticercoids of Flamingolepis liguloides, Cestode of the Flamingo (Phoenicopterus ruber) shows the presence of three other cysticercoids of cestode parasites of the Flamingo in the hemocoele of the Branchiopode.

A fourth one is reported as the cysticercoid of Eurycestus avoceti, Clark, 1954, which parasitizes the Avocet (Recurvirostra avosetta). The systematic position of this Cestode is always unknown.

This report shows the importance of Artemia in the life cycle of Cestodes of Anseriforms and Charadriiforms birds in saline lagoons.

* En l'absence de caractérisation biochimique, il est recommandé de ne plus employer Artemia salina et de désigner les populations par Artemia sp. seul valable du point de vue taxonomique (recommandation des participants au Symposium International sur Artemia salina réunis à Corpus christi, Texas, U.S.A., 20-23 août 1979).

Accepté le 16 janvier 1979. 
Au cours d'une étude sur la prévalence des larves de Flamingolepis liguloides (Cestode Hymenolepididae) parasite du Flamant (Phoenicopterus ruber) dans les populations d'Artemia sp. des salines de Camargue, nous avons observé dans l'hémocoele de ces Branchiopodes (outre $F$. liguloides) quatre autres cysticercoïdes de Cestodes Cyclophyllides.

D'après le nombre et la morphologie des crochets, nous rapportons trois de ces cysticercoïdes à des Cestodes de Flamant :

- F. famingo (Skrjabin, 1914) Spassky et Spasskaja, 1954 (8 crochets de $58 \mu \mathrm{m})$,

- F. caroli Parona, 1887 (8 crochets de 110 à $130 \mu \mathrm{m})$,

- et Gynandrotaenia stammeri Fuhrmann, 1936 ( 6 crochets de $50 \mu \mathrm{m}$ et ventouses armées de plusieurs rangées de petites épines) dont Gvozdev et Maksimova (1979) viennent de réaliser le cycle chez Artemia sp. en Russie.

Le quatrième ( $f$ ig. 1 et $3 \mathrm{~A})$ est un cysticercoïde de petite taille $(170 \times 90 \mu \mathrm{m})$ encapsulé dans le thorax et repérable à la coloration marron-fonçé de la capsule réactionnelle.

A l'intérieur de la cavité d'invagination, le scolex dont le diamètre est de $70 \mu \mathrm{m}$ est muni de 4 ventouses de $20 \mu \mathrm{m}$ de diamètre (fig. 1 et $3 \mathrm{~A}$ ). Les ventouses sont armées sur les bords antérieur et latéraux de 2 rangées de 15 à 16 petites épines de $7 \mu \mathrm{m}$ de longueur (fig. $3 \mathrm{~B}$ ). Le rostre est muni de 16 crochets disposés en deux couronnes alternées. Les 8 petits mesurent $12 \mu \mathrm{m}$, les 8 grands $18 \mu \mathrm{m}$ (fig. 2 et $3 C, D$ ).

Nous rapportons ce cysticercoïde à Eurycestus avoceti Clark, 1965, dont l'adulte a été signalé par Baer (1968) chez l'Avocette (Recurvirostra avosetta) en Camargue. En effet, les caractères du scolex, en particulier la double couronne de crochets rostraux et la présence de ventouses armées d'épines sur les bords antérieur et latéraux sont en tout point semblables. La seule différence par rapport à la description de Baer réside dans le nombre d'épines ventousaires (de 30 à 32 au lieu de 16 à 18).

L'adulte de Eurycestus avoceti a été observé pour la première fois par Clark (1954) chez une Avocette américaine (Recurvirostra americana). D'après la description de Clark, la caractéristique la plus originale de ce Cestode, outre la grande extension des champs latéraux de chaque segment, est la complète duplication de l'appareil génital. N'ayant pu observer le scolex, Clark a préféré ne pas attribuer cette espèce à une famille précise de Cyclophillidea mais estimait, du fait de la présence d'un appareil double, qu'il s'agissait d'un Davaneidae proche du genre Cotugnia.

Yamaguti (1959), à partir de la description de Clark place E. avoceti parmi les Anoplocephalidae, sans raison apparente, le scolex n'ayant pas été décrit.

Baer (1968) retrouve chez l'Avocette de Camargue, un Cestode dont l'anatomie rappelle celle d'E. avoceti. La présence du scolex et des premiers segments permet à l'auteur d'observer une double couronne de crochets rostraux et quatre ventouses armées d'épines et de préciser qu'il n'y a pas duplication des organes génitaux mais alternance régulière d'un segment à l'autre, ce caractère n'étant apparent que dans 


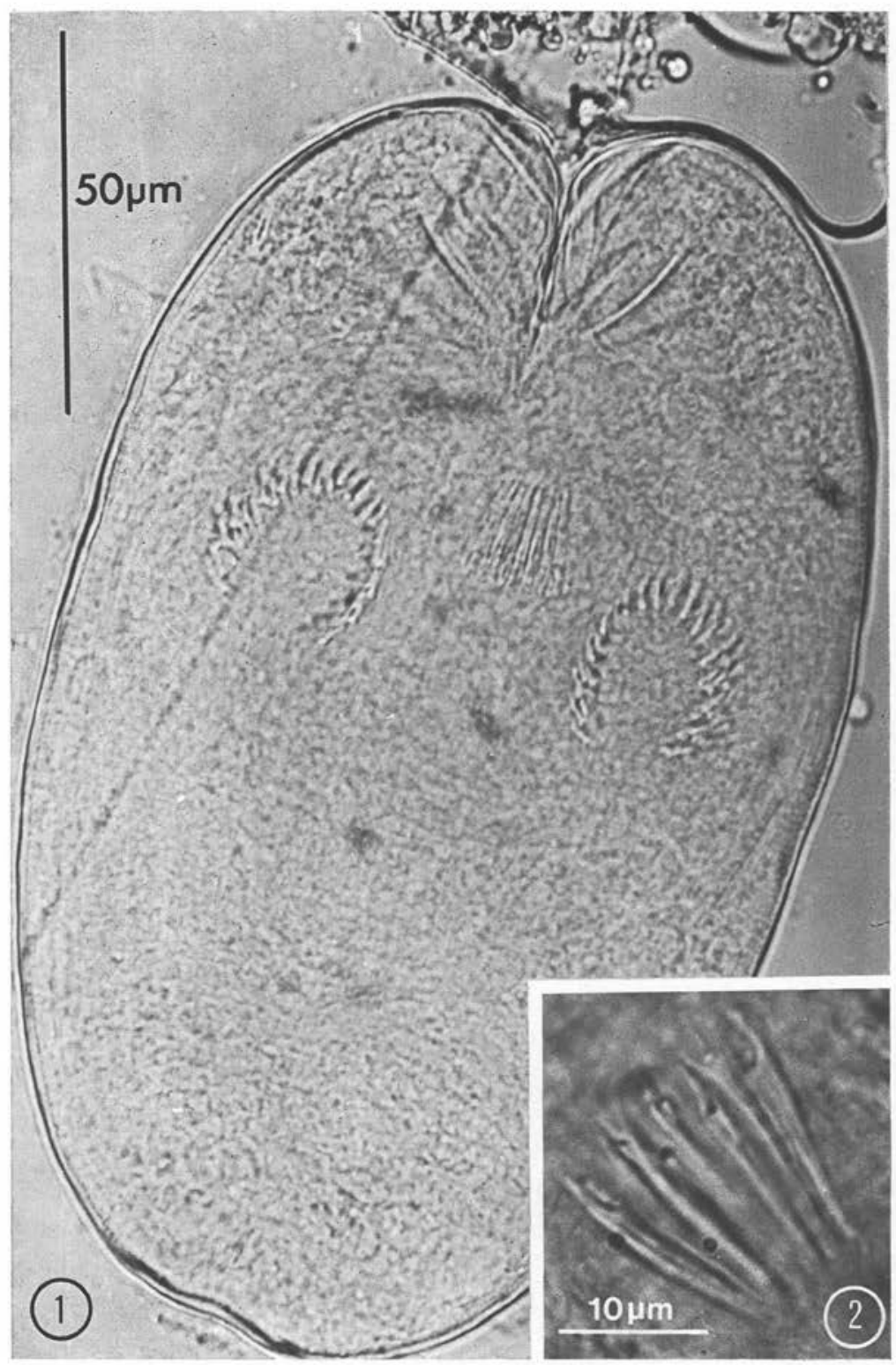

Fig. 1. Cysticercoïde d'Eurycestus avoceti.

Fig. 2. Crochets du rostre. 


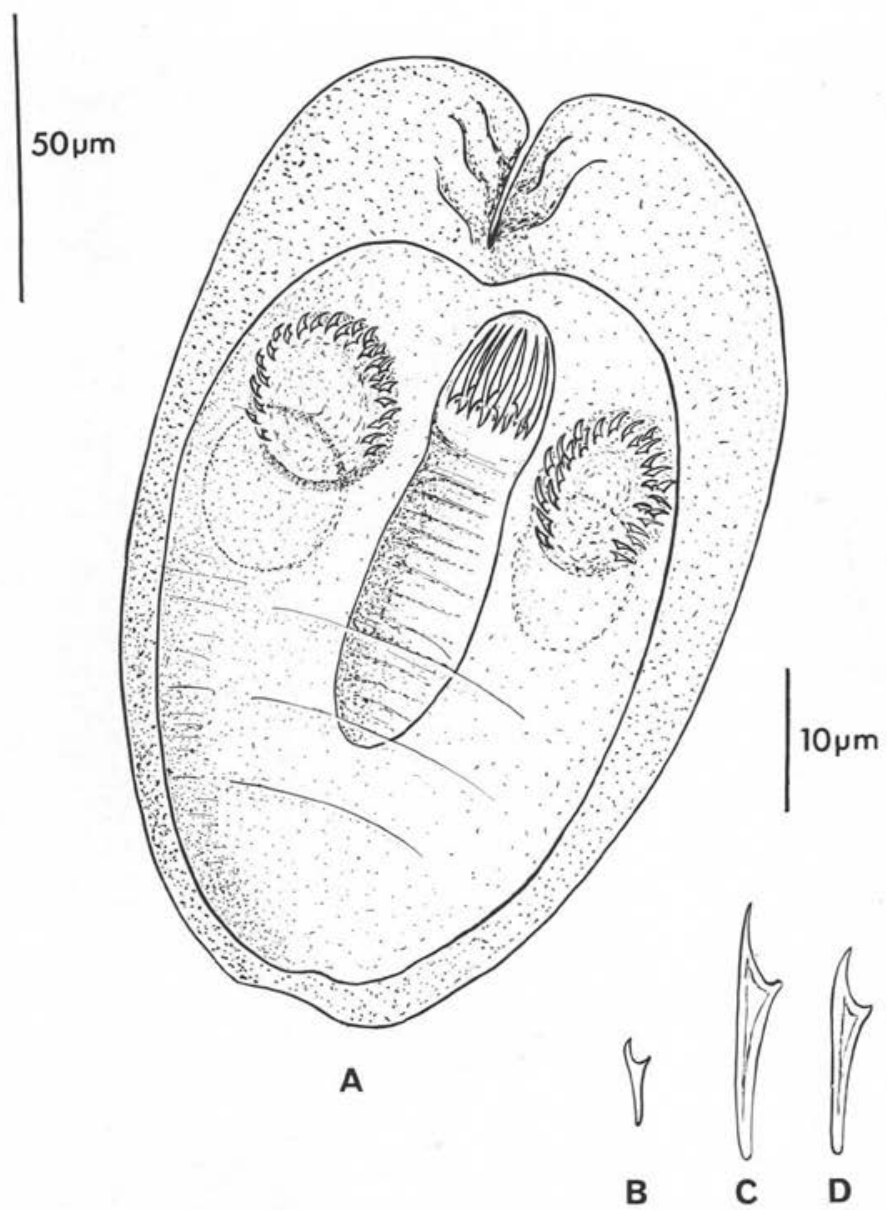

Fig. 3. Cysticercoïde d'Eurycestus avoceti. A : Vue d'ensemble du cysticercoïde; B : Epine ventousaire. $\mathrm{C}$ et $\mathrm{D}$ : Crochets du rostre.

les jeunes proglottis. Ces caractéristiques, ainsi que la présence d'un vagin l'amènent à classer $E$. avoceti parmi les Dilepididae.

Les premières observations du cysticercoïde d'E. avoceti qui font l'objet de ce travail n'apportent aucun argument pour déterminer la place systématique de ce Cestode. Seule, la réalisation expérimentale du cycle permettrait, en précisant l'anatomie de l'adulte et l'ontogénèse du cysticercoïde de résoudre cette question.

La découverte chez Artemia d'un Cysticercoïde de Cestode parasite de l'Avocette et de trois cysticercoïdes de Cestodes de Flamant en Camargue, après celle de cysticercoïdes de Flamants (Maksimova, 1973), de Courlis (Maksimova, 1973), de Tadornes 
(Maksimova, 1976) et de Laridae (Codreanu et Codreanu-Balcescu, 1978) dans divers lacs salés d'Europe montre l'importance de ce Branchiopode dans les cycles des Cestodes d'Oiseaux (Lariformes, Ansériformes, Charadriiformes) fréquentant les milieux lagunaires salés.

\section{Bibliographie}

Baer J.G.: Eurycestus avoceti Clark, 1954 (Cestode Cyclophyllidien) parasite de l'Avocette en Camargue. Vie, Milieu, 1968, 19, 189-198.

Clark D. T. : A new Cyclophyllidean Cestode from the Avocet. J. Parasitol., 1954, 40, 340-346.

Codreanu R., Codreanu-Balcescu D.: The occurrence in Artemia salina L. (Crustacea Anostracea) from Romania of a peculiar cysticercoid larva belonging to avian Hymenolepididae (Cestodes). $4^{e}$ Congrès International de Parasitologie, 1978, Varsovie, section B, p. 39.

Gvozdev E. V., Maksimova A.P.: Morphologie et cycle évolutif du Cestode Gynandrotaenia stammeri (Cestoïdea, Cyclophyllidea) parasite de Phoenicopterus roseus. Parazitologiya SUN, 1979. 13, 56-60.

Maksimova A.P.: Branchiopoda as the intermediate hosts of Hymenolepididae. Materials of the International conference on Hymenolepididae, Varsovie, 14-16 septembre 1973, pp. 82-85.

Maksimova A.P.: A new Cestode, Fimbriarioides tadornae sp. n., from Tadorna tadorna and its development in the intermediate host. Parazitologiya, 1976, 10, 16-24.

Yamaguti S. : Systema helminthum Volume II. The Cestodes of Vertebrates, 860 pages. Interscience, Publ., New York, 1959, 860 p. 